\title{
In-flight scalar calibration and characterisation of the Swarm magnetometry package
}

\author{
Lars Tøffner-Clausen ${ }^{1 *} \mathbb{D}$, Vincent Lesur ${ }^{2}$, Nils Olsen ${ }^{1}$ and Christopher C. Finlay ${ }^{1}$
}

\begin{abstract}
We present the in-flight scalar calibration and characterisation of the Swarm magnetometry package consisting of the absolute scalar magnetometer, the vector magnetometer, and the spacecraft structure supporting the instruments. A significant improvement in the scalar residuals between the pairs of magnetometers is demonstrated, confirming the high performance of these instruments. The results presented here, including the characterisation of a Sun-driven disturbance field, form the basis of the correction of the magnetic vector measurements from Swarm which is applied to the Swarm Level $1 \mathrm{~b}$ magnetic data.
\end{abstract}

Keywords: Geomagnetism, Magnetometer, Instrument calibration, Satellite, Swarm

\section{Introduction}

In November 2013 the European Space Agency (ESA) launched the three Swarm satellites, named Alpha, Bravo, and Charlie, with the objective to provide the best ever survey of the geomagnetic field and its temporal evolution (Friis-Christensen et al. 2006). Each spacecraft carries an Absolute Scalar Magnetometer (ASM) for measuring Earth's magnetic field intensity, a Vector Fluxgate Magnetometer (VFM) measuring the direction and strength of the magnetic field, and a three-head Star TRacker (STR) mounted close to the VFM to obtain the attitude needed to transform the vector readings to an Earth-fixed coordinate frame. Time and position are provided by an on-board GPS receiver. The payload also includes instruments to measure plasma and electric field parameters as well as non-gravitational acceleration.

One of the purposes of the scalar magnetometer (ASM) is to provide the necessary absolute magnetic data to calibrate the vector magnetometer (VFM). For this an approach similar to that adopted for the previous satellite missions Ørsted and CHAMP was foreseen (c.f. Olsen

\footnotetext{
*Correspondence: lastec@space.dtu.dk

1 Division of Geomagnetism, DTU Space, Technical University of Denmark, Diplomvej, Kongens Lyngby, Denmark

Full list of author information is available at the end of the article
}

2003; Yin and Lühr 2011) since those missions carried equivalent instrumentation. However, soon after launch of Swarm it became clear that the magnetic field vector measurements on all three spacecraft were contaminated by unforeseen disturbances which could not be captured by the traditional in-flight calibration methods referred to above. Furthermore, the disturbances show systematic variation which could impact or map into scientific investigations based on Swarm magnetic data. The light blue symbols in Fig. 1 show time series of the scalar residuals, which are the difference, $\Delta F=\left|\vec{B}_{\mathrm{VFM}}\right|-F_{\mathrm{ASM}}$, between the modulus of the VFM data, $\left|\vec{B}_{\mathrm{VFM}}\right|$, and the magnetic intensity measurements, $F_{\mathrm{ASM}}$, taken by the ASM instrument. Based on experience with Ørsted and CHAMP scalar residuals with sub-nanotesla level were expected (rms value well below $0.5 \mathrm{nT}$ ), while for Swarm the scatter of the residuals was observed to reach several $\mathrm{nT}$, resulting in an rms value approaching $1 \mathrm{nT}$, but crucially showing a very clear local time dependence. A task force was therefore established to investigate and mitigate the effect.

Detailed investigations of the scalar residuals $\Delta F$ and of the ASM and VFM measurements separately indicated that:

- the vector readings of the VFM are affected by a disturbance vector field; 


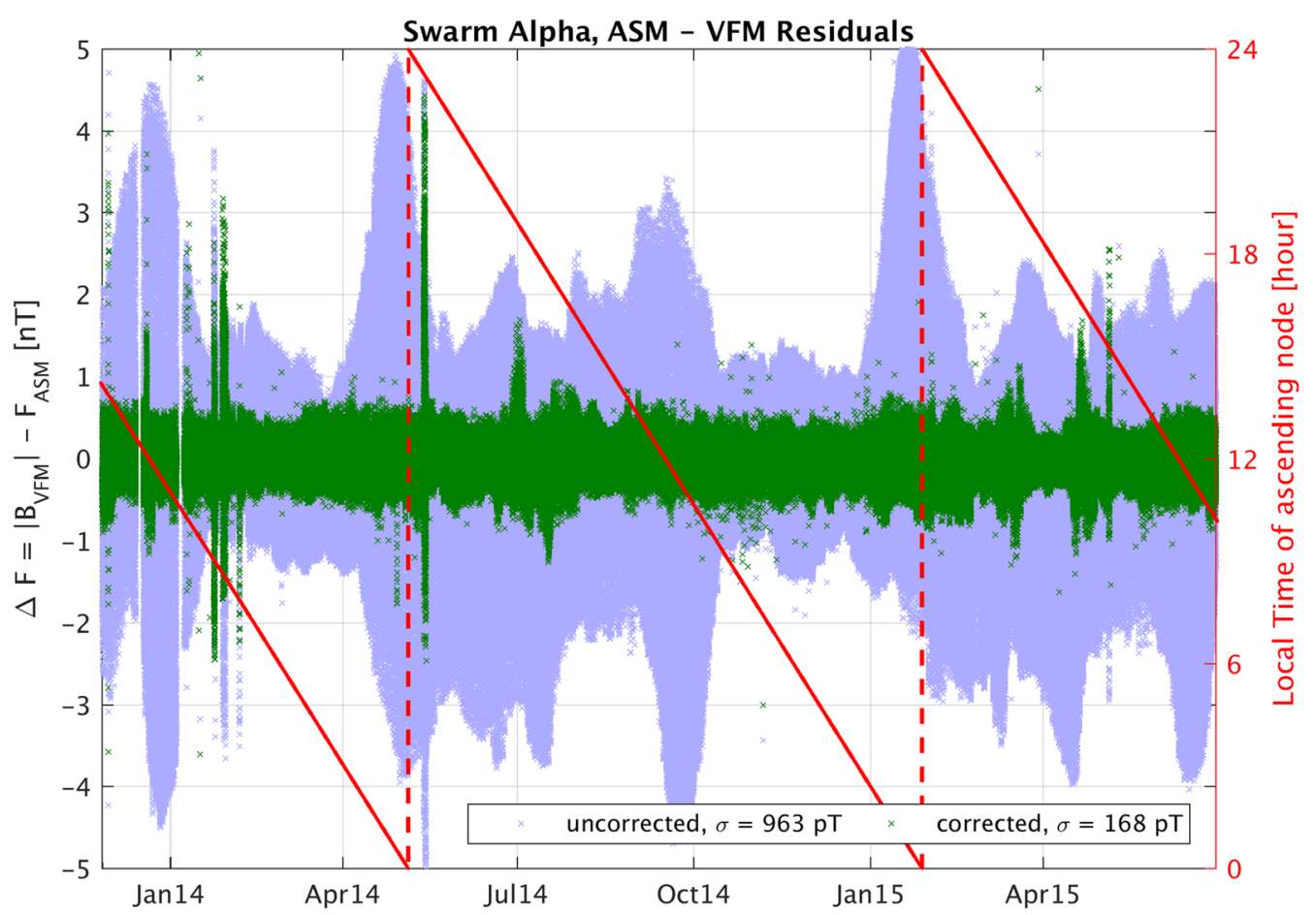

Fig. 1 Scalar residuals of uncorrected (light blue) and corrected (green) measurements versus time. Local time of the ascending node is shown in red (right axis)

- the scalar readings of the ASM are much less, if at all, affected.

Consequently, the task force concluded to pursue models which assume the magnetic disturbance to be affecting the VFM measurements only. Plotting $\Delta F$ as a function of the Sun incidence angles with respect to the spacecraft, reveals systematic features of the disturbance, as shown in Fig. 2. At the start of "Characterisation and calibration with scalar residuals" section we provide detailed definitions of the two Sun incidence angles $\alpha$ and $\beta$. This supports the hypothesis that a magnetic source in the vicinity of the VFM magnetometer, with strength and direction depending on the direction to the Sun (as seen from the spacecraft), is responsible. We refer to such a disturbance field vector that depends on the direction to the Sun, as $\delta \vec{B}_{\text {Sun }}$.

The purpose of this article is to document the details of in-flight calibration of the Swarm magnetometer package, including an empirical determination and removal of the Sun-driven vector disturbance field $\delta \vec{B}_{\text {Sun }}$, based on a mitigation approach proposed by Vincent Lesur (Lesur et al. 2015).

"Characterisation and calibration with scalar residuals" section describes the parameterisation of the model of the Sun-driven disturbance-in following referred to as the characterisation of the disturbance field-and of the calibration of the VFM instrument, by which means determination of its intrinsic scale factors and their dependence on time and temperature, and determination of the sensor-axis non-orthogonalities. We document the adopted Iteratively Reweighted Least Squared (IRLS) estimation approach that includes a truncated singular value decomposition (SVD) approach to solving the inverse problem. The results obtained for Swarm Alpha, based on data covering the period from launch (22 November 2013) until end of June 2015 (i.e. 19 months), are presented in "Results of model estimation for Swarm Alpha" section. Application of the scheme to data from the satellites Bravo and Charlie resulted in similar levels of residual improvement and statistics, and the estimates of the Sun-driven disturbance $\delta \vec{B}$ Sun show generally similar behaviour and structural features as found for Swarm Alpha, although there are also some differences. Finally, "Conclusions" section summarises the findings and provides perspectives regarding further improvements of the method.

\section{Characterisation and calibration with scalar residuals}

The Sun incidence angles $\alpha$ and $\beta$ are crucial in our approach to characterise the scalar residual. To clarify, 


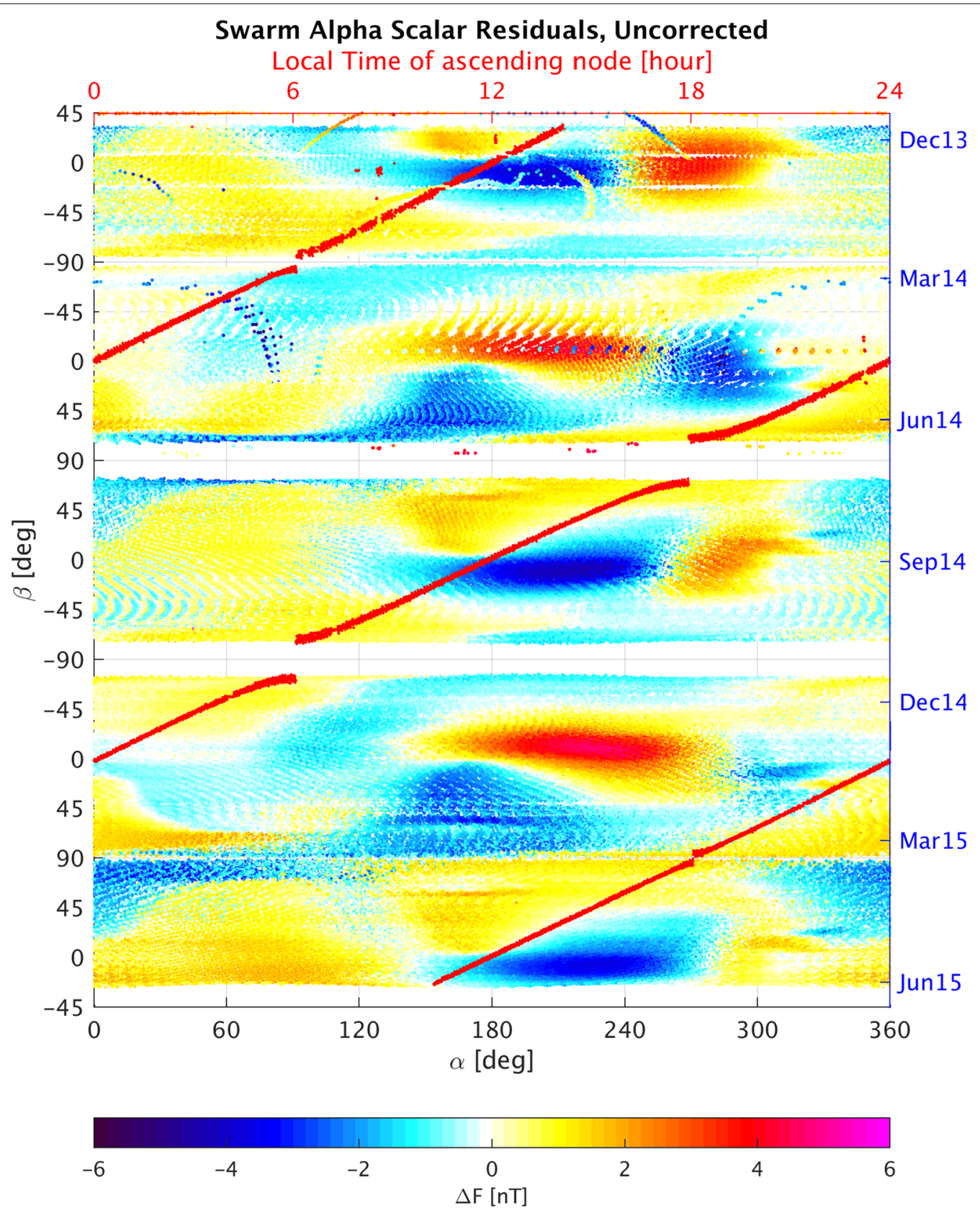

Fig. 2 Uncorrected scalar residuals between ASM and VFM magnetometers, $\Delta F$, plotted versus Sun incident angles $\alpha$ and $\beta$. The $\beta$ angle oscillates slowly in time; hence, the un-folded $\beta$ angle corresponds to season which is indicated in blue on the right-hand side. Local time of the ascending node is shown in red (axis on top)

in Fig. 3 we illustrate the definition of these angles with respect to the spacecraft and the Sun position. $\alpha$ is the azimuth in the spacecraft $x-z$ plane (nominally the orbit plane), and $\beta$ is the "elevation" out of the $x-z$ plane positive towards left (looking in the nominal flight direction; i.e. positive opposite the spacecraft $y$-axis). Examples of values for $\alpha$ and $\beta$ for particular Sun positions are:
- $\beta=+90^{\circ}$ : Sun directly from $-y$ (i.e. from the left during nominal flight)

- $\beta=-90^{\circ}$ : Sun directly from $+y$ (i.e. from the right)

- $\beta=0^{\circ}, \alpha=0^{\circ}$ : Sun directly from $+x$ (i.e. from the front)

- $\beta=0^{\circ}, \alpha=+90^{\circ}$ : Sun directly from $-z$ (above)

- $\beta=0^{\circ}, \alpha=+180^{\circ}$ : Sun directly from $-x$ (i.e. from the back-slightly above the boom) 


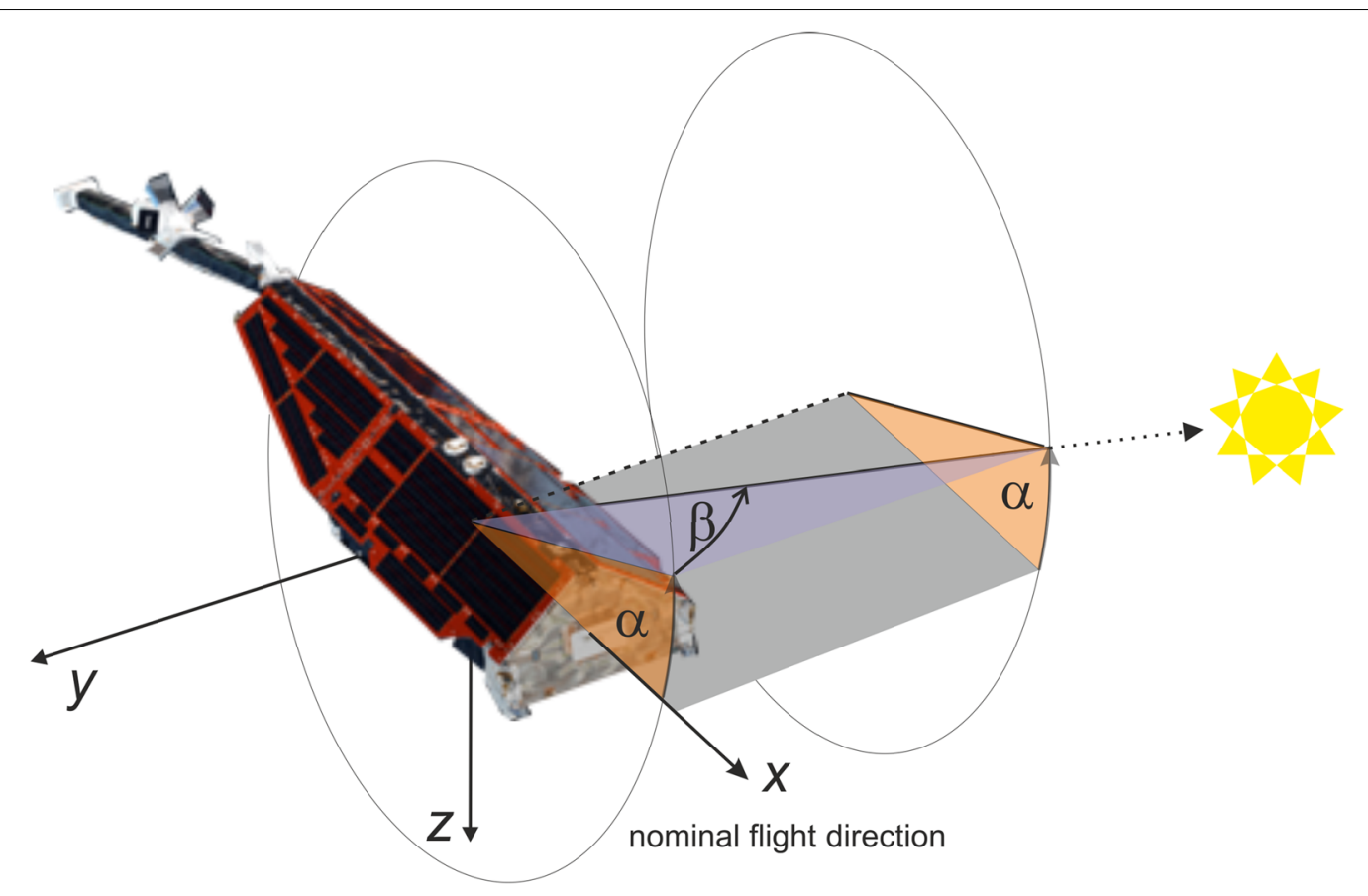

Fig. 3 Illustration of Sun incident angles $\alpha$ and $\beta$ that are defined w.r.t. the spacecraft. $\alpha$ is the "azimuth" from $x$ about the $y$-axis, and $\beta$ is the "elevation" from the $x-z$ plane

Considering how these angles vary over orbits of the Swarm spacecraft during nominal flight, we find that $\alpha$ varies rapidly from $360^{\circ}$ down to $0^{\circ}$ within one orbit (i.e. within $\approx 90 \mathrm{~min}$ ), while $\beta$ varies slowly up and down typically by $\approx 1.25^{\circ}$ in one day (for Alpha and Charlie, $1.20^{\circ}$ for Bravo).

Although the observed scalar residuals clearly vary with the Sun incidence angles $\alpha$ and $\beta$ (see Fig. 2), there is no direct mapping of $\Delta F$ in terms of these parameters. This is a consequence of the scalar residuals $\Delta F \approx \delta \vec{B}_{\text {Sun }} \cdot \vec{b}_{0}$ being the projection of the magnetic disturbance vector $\delta \vec{B}_{\text {Sun }}$, onto the unit vector $\vec{b}_{0}$ of the ambient magnetic field direction (Earth's main field). The former is oriented relative to the spacecraft, while the latter is oriented relative to Earth, which results in the variations with the spacecraft local time (captured by $\beta$ ) as seen in Fig. 2. The spacecraft local time changes by 12 hours (corresponding to a change in $\beta$ by $180^{\circ}$ ) within approximately $4 \frac{1}{2}$ months.

To account for the projection on to the ambient field, we consider a vector magnetic disturbance $\delta \vec{B}_{\text {Sun }}(\alpha, \beta)$, with each component depending individually on the Sun incidence angles. Mathematically, we describe each component of the disturbance field vector by a spherical harmonic expansion in $\alpha$ and $\beta$ i.e. we consider three independent spherical harmonic expansions in all.
This model characterising the Sun-driven disturbance is co-estimated together with a model of the temporal evolution of the VFM sensitivity and an adjustment of the pre-flight estimated non-orthogonality angles of the VFM sensor. For this we perform a scalar calibration via a least squares fit, minimising the discrepancy $(\Delta F)$ between the fully calibrated and corrected measurements from the ASM and the modulus of the vector measurements from the VFM after our model has been applied. Huber weights are used iteratively to eliminate the effect of anomalous measurements ("outliers") on the estimated models.

\section{Model parameterisation}

As outlined above, our model characterising the Sundriven disturbance vector $\delta \vec{B}_{\text {Sun }}$ consists of three spherical harmonic expansions up to degree and order 25 , one for each of the magnetic field components in the VFM magnetometer frame, with the position of the Sun with respect to the spacecraft parameterised by the Sun incidence angles $\alpha$ and $\beta$. It takes the form

$$
\delta \vec{B}_{\text {Sun }}=\sum_{n=0}^{25} \sum_{m=0}^{n}\left(\vec{u}_{n}^{m} \cos m \alpha+\vec{v}_{n}^{m} \sin m \alpha\right) P_{n}^{m}(\sin \beta)
$$


where $\vec{u}_{n}^{m}$ and $\vec{v}_{n}^{m}$ are the spherical harmonic expansion coefficients, with one component for each component of the disturbance field, and $P_{n}^{m}$ are the Schmidt semi-normalised Legendre functions. Note that $\delta \vec{B}_{\text {Sun }}$ includes static terms $(n=m=0)$ that describe a static (i.e. independent of the Sun position) disturbance vector. The disturbance field vector $\delta \vec{B}_{\text {Sun }}$ is thus described by $3 \times 26^{2}=2028$ model coefficients.

The model for re-scaling the vector measurements and taking into account any small adjustment of the nonorthogonality of the VFM sensors, which is required in order to obtain the fully calibrated and corrected vector field measurements $\vec{B}_{\mathrm{VFM}}$, now takes the form

$$
\vec{B}_{\mathrm{VFM}}=\underline{\underline{P}}^{-1} \underline{\underline{S}}^{-1} \vec{B}_{\text {pre-flight }}-\delta \vec{B}_{\text {Sun }}
$$

where $\vec{B}_{\text {pre-flight }}$ are the VFM measurements calibrated using the pre-flight parameters and corrected for the pre-flight determined stray fields as described in TøffnerClausen (2015). $\underline{\underline{S}}$ is a $3 \times 3$ diagonal scaling matrix with elements

$$
s_{j}=s^{\mathrm{B}-\text { spline }}(t)+s_{j, \text { Tsensor }} T_{\text {sensor }}+s_{j, \beta} \beta
$$

where $s^{\mathrm{B} \text {-spline }}(t)$ is a quadratic B-spline in time with 3 -month knot separation (common for all three components of the magnetic field), and $s_{j \text {,Tsensor }}, j=1-3$ is an adjustment of the pre-flight estimated dependency of the VFM sensitivity on its sensor temperature, $T_{\text {sensor }}$, for each sensor axis $j . s_{j, \beta}$ is an empirical scaling parameter and $\beta$ the Sun incidence angle, as defined above. The choice of quadratic B-splines with 3-month knot separation is made to allow sufficient flexibility of the model; the exact choice of B-spline knot times is not crucial as very similar results are obtained with other, similar parameterisations. The estimated B-splines exhibit very moderate accelerations (in the case of the full model, see Fig. 6), and it may be possible to simplify the parameterisation of the time dependence in future models, e.g. to an exponential saturation in time as this is the expected behaviour of the VFM instrument sensitivity; however, an exponential model is ill-conditioned on the time span of data used here.

$\underline{P}$ is the non-orthogonality matrix that makes small adjustments to the pre-flight estimated non-orthogonalities of the VFM sensor (cf. Olsen 2003)

$\underline{\underline{P}}=\left(\begin{array}{ccc}1 & 0 & 0 \\ -\sin u_{1} & \cos u_{1} & 0 \\ \sin u_{2} & \sin u_{3} & \sqrt{1-\sin ^{2} u_{2}-\sin ^{2} u_{3}}\end{array}\right)$

Our in-flight calibration model comprises 18 parameters in all; together with the 2028 parameters describing $\delta \vec{B}_{\text {Sun }}$ this results in 2046 model parameters to be estimated, as listed in Table 1.

\section{Estimation of model parameters: inversion and regularisation}

In order to estimate the 2046 model parameters from the scalar residuals we need to solve a nonlinear inverse problem. The nonlinearity arises from the treatment of non-orthogonalities (Olsen 2003).

The forward relationship between the vector of the scalar residuals, $\mathbf{d}\left(d_{i}=\Delta F_{i}\right.$, the scalar residual of the $i$ th data point), and the model parameter vector $\mathbf{m}$, may therefore be written in the form

$$
\mathbf{d}=\mathbf{g}(\mathbf{m})+\mathbf{e}
$$

where $\mathbf{g}(\mathbf{m})$ is a nonlinear function of the model parameters and $\mathbf{e}$ is a small remainder that cannot be explained by the model, which we seek to minimise.

Linearisation of this problem is straightforward. A regularised, iteratively reweighted, least squares solution to the inverse problem is then obtained using the algorithm

$$
\begin{aligned}
\mathbf{m}_{k+1}= & \mathbf{m}_{k}+\left(\underline{\underline{\mathbf{G}}}_{k}^{T} \underline{\underline{\mathbf{W}}}_{k} \underline{\underline{\mathbf{G}}}_{k}+\lambda \underline{\underline{\mathbf{R}}}^{-1}\right. \\
& \left(\underline{\underline{\mathbf{G}}}_{k}^{T} \underline{\underline{\mathbf{W}}}_{k}[\mathbf{d}-\mathbf{g}(\mathbf{m})]-\lambda \underline{\underline{\mathbf{R}}} \mathbf{m}_{k}\right)
\end{aligned}
$$

where at the $k$ th iteration, $\underline{\underline{\mathbf{G}}}_{k}=\left.\frac{\partial \mathbf{g}(\mathbf{m})}{\partial \mathbf{m}}\right|_{\mathbf{m}=\mathbf{m}_{k}}$, is the appropriate Jacobian matrix, $\underline{\underline{\mathbf{R}}}$ is a regularisation matrix discussed in detail below, and $\underline{\underline{\mathbf{W}}}_{k}$ is a (Huber) weighting matrix.

$\underline{\underline{\mathbf{W}}}_{k}$ is updated at each iteration and consists of diagonal elements

$$
{ }^{k} w_{i}=\min \left(1, \frac{c \sigma}{{ }^{k} d_{i}}\right) .
$$

${ }^{k} d_{i}$ is the scalar residual of the $i$ th data point using model vector $\mathbf{m}_{k}$ and

$$
\sigma=\sqrt{\frac{\sum_{i}\left({ }^{k-1} w_{i}{ }^{k} d_{i}\right)^{2}}{\sum_{i}\left({ }^{k-1} w_{i}\right)^{2}}}
$$

Table 1 Model parameters

\begin{tabular}{llc}
\hline Description & Parameters & Dimension \\
\hline$\delta \vec{B}_{\text {Sun }}$ & $\vec{u}, \vec{v}$ & 2028 \\
Sensitivity, time dependent & $s^{\text {Bspline }}$ & 9 \\
Sensitivity, $\beta$ dependency & $\vec{s}_{\beta}$ & 3 \\
Sensitivity, sensor temperature dependency & $\vec{s}_{\text {Tsensor }}$ & 3 \\
Non-orthogonalities & $u_{1}, u_{2}, u_{3}$ & 3 \\
Total & & 2046 \\
\hline
\end{tabular}


being a (robust) estimate of the standard deviation of the residuals at iteration $k$. We set $c=2$, slightly higher than the value of 1.5 usually chosen, in order to ensure that the less numerous polar data are not overly downweighted in the determination of the calibration parameters.

It turns out that the full set of 2046 parameters is not needed to obtain good results and low data misfit, which is confirmed by inspection of the eigenvalues of the matrix $\left(\underline{\underline{\mathbf{G}}}_{k}^{T} \underline{\underline{\mathbf{W}}}_{k} \underline{\underline{\mathbf{G}}}_{k}+\lambda \underline{\underline{\mathbf{R}}}\right)$, as presented in Fig. 4 for Swarm Alpha. The magnitudes of the sorted eigenvalues (in order of decreasing magnitude) exhibit a distinct drop around 750-800 degrees of freedom, indicating the smaller eigenvalues contribute little to the solution. The inversion of this matrix was therefore finally performed using a truncated singular value decomposition (TSVD) procedure, retaining only 750 degrees of freedom.

A regularisation matrix $\underline{\underline{\mathbf{R}}}$ is also included to help stabilise the inversion. This is necessary because the Swarm satellites operate in a tightly controlled attitude orientation which leads to a poor excitation of the VFM instrument along the axis perpendicular to the orbit plane (the east-west direction corresponding to the $y$-axis of the VFM sensor). Consequently, the parameters related to the $y$-axis are poorly determined in a scalar calibration. The regularisation matrix $\underline{\underline{\mathbf{R}}}$ is therefore defined so that it acts on the parameters $s_{2}$,Tsensor, $s_{2, \beta}, u_{1}$, and $u_{3}$ to force $s_{2, \text { Tsensor }} \simeq\left(s_{1, \text { Tsensor }}+s_{3, \text { Tsensor }}\right) / 2$ (to reflect the physical properties of the VFM sensor) and also to minimise the norms $s_{2, \beta}^{2}$ and $u_{1}^{2}+u_{3}^{2}$. $\lambda$ is chosen to be sufficiently large to effectively impose the regularisation on the estimated model. Note that no regularisation is directly imposed on $\delta \vec{B}_{\text {Sun }}$ but use of truncated SVD during the inversion automatically acts to suppresses structure in regions that are not well constrained by the input data.

The starting model for the inversions is "unity", i.e. $\underline{P}=\underline{S}=\underline{I}$, where $\underline{I}$ is the identity matrix, and $\vec{u}_{n}^{m}=\vec{v}_{n}^{m}=\overrightarrow{0}$. The inversions typically converge within 25 iterations.

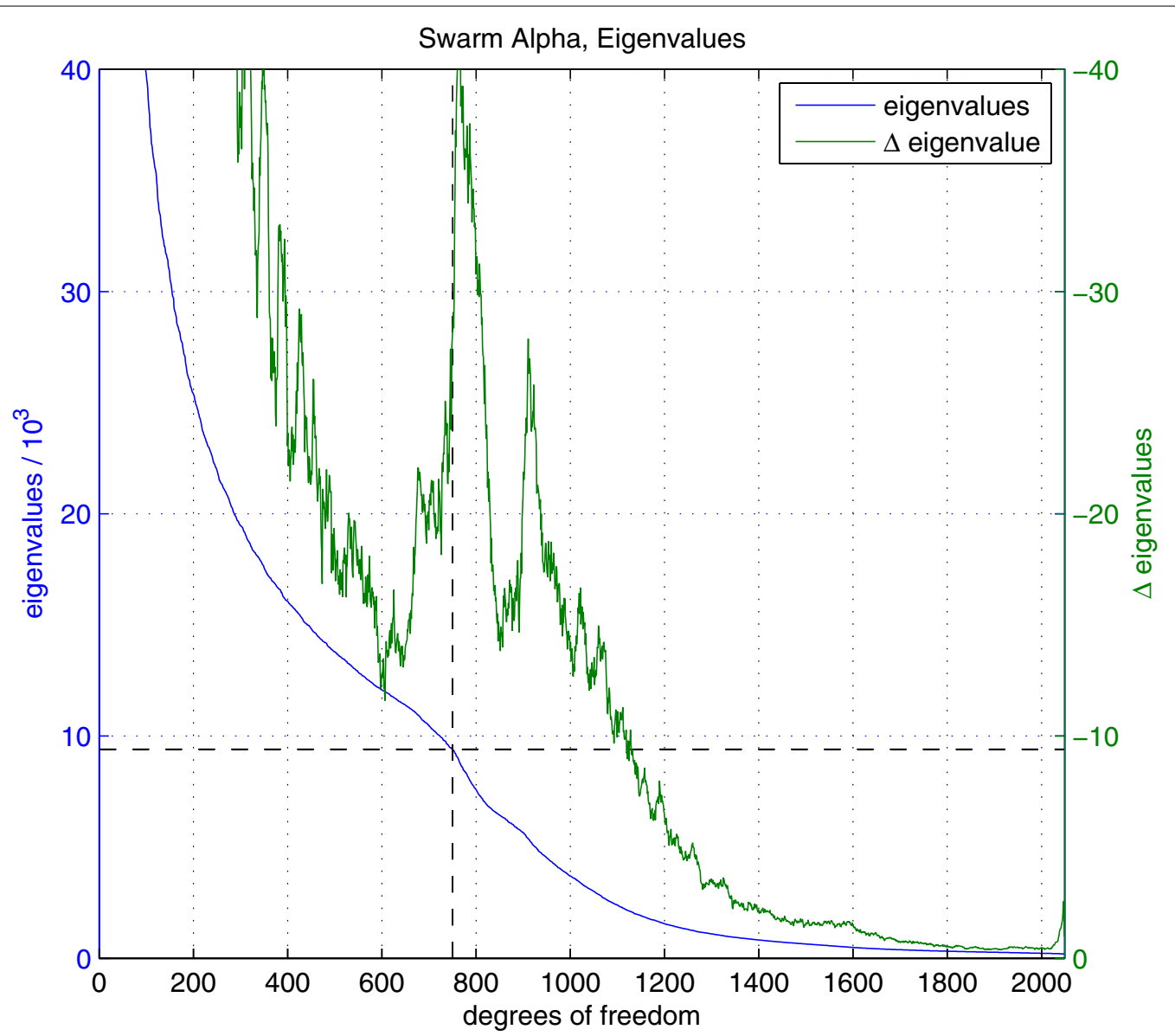

Fig. 4 Eigenvalues of $\underline{\underline{G}}^{T} \underline{\mathbf{G}}+\lambda \underline{\underline{\mathbf{R}}}$ matrix (blue, left axis), sorted decreasingly. In green (right axis), the decrease per eigenvalue is plotted 


\section{Results of model estimation for Swarm Alpha}

The model described above is estimated for Swarm Alpha using data from the beginning of the mission (22 November 2013) until June 2015. Figure 1 shows the final scalar residuals, i.e. the residuals after application of the model (after "calibration and correction") of the VFM measurements (in green), as a function of time together with the residuals of the un-corrected but re-scaled vector field measurements, i.e. $\vec{B}_{\mathrm{VFM}}+\delta \vec{B}_{\text {Sun }}$, in light blue; these data illustrate what can be achieved with the traditional scalar calibration methods. Note the excellent reduction of the scalar residuals achieved by the model; the Huber weighted rms of the residuals drops from 963 to $168 \mathrm{pT}$. Table 2 provides the corresponding numbers for Bravo and Charlie.

Figure 5 shows normal distribution plots for the scalar residuals. The top plot shows the distributions of all data for un-corrected (red) and fully corrected data (green) and demonstrates a transition from a non-Gaussian to Gaussian residual distribution when applying the model. The bottom plots show the distributions of the data split into 3-month periods, un-corrected to the left and corrected to the right. These also demonstrate the elimination of systematic and non-Gaussian effects.

Table 3 lists the estimated $s_{\text {Tsensor }}$ and $s_{\beta}$ parameters and the non-orthogonality values for all three Swarm satellites together with their estimated pre-flight values for the VFM instrument itself for reference. I.e. the table shows the adjustments applied in order to reduce the scalar residuals to the level indicated above.

Table 4 shows the increase in the weighted rms of the scalar residuals when omitting individual parts of the model-a full re-estimation of the remaining model

Table 2 Scalar residual statistics, uncorrected, and corrected data

\begin{tabular}{lll}
\hline Satellite & \multicolumn{2}{l}{ Weighted rms (pT) } \\
\cline { 2 - 3 } & Uncorrected & Corrected \\
\hline Alpha & 962.6 & 168.3 \\
Bravo & 710.3 & 164.2 \\
Charlie & & \\
FASM & 632.1 & 172.3 \\
FAC,map & 862.1 & 527.7 \\
\hline
\end{tabular}

For Swarm Charlie two sets of numbers are given: one set for which the ASM was still working $\left(F_{\mathrm{ASM}}\right.$, until 5 November 2014$)$ and one set using the scalar data from Swarm Alpha mapped to the position of Swarm Charlie $\left(F_{\mathrm{AC} \text {,map }}\right)$. For data from 1 May 2014 through 5 November 2014 the weighted rms of $F_{\mathrm{ASM}}-F_{\mathrm{AC} \text {,map }}$ is 572.6 pT parameters is carried out for each table entry. Particularly the omission of the non-orthogonalities drastically increases the misfit-the power (the mean-square) is more than doubled. Due to the stable attitude of the Swarm satellites, the small $x-z$ non-orthogonality angle, $u_{2}$, is equivalent to first order to a small, relative timeshift between the ASM and VFM measurements -1 arc-second corresponds roughly to a 3-ms timeshift, and it has been discussed whether it would be more reasonable to introduce such timeshifts rather than adjusting the preflight estimated non-orthogonalities. However, the variations in the $u_{2}$ angles estimated by this model would imply time-shifts varying from $-3 \mathrm{~ms}$ to $+13 \mathrm{~ms}$ for the individual satellites which, to the authors, seems quite unlikely.

The temporal evolution of the scaling of the vector field measurements, $s^{\mathrm{B} \text {-spline }}$, is shown in Fig. 6 for the various test models listed in Table 4. The full model, shown in red, shows a smooth behaviour in time, as expected from an instrument design perspective. The blue curve shows the model without $s_{\beta}$; this exhibits some small oscillations, whereas the light brown (no $s_{\text {Tsensor }}$ ) and green (no $\delta \vec{B}_{\text {Sun }}$ ) curves show much higher level of oscillations indicating they are inadequate to capture the behaviour of the measurements. The elimination of the oscillations in the full model is a good indicator of the validity of this model. The magenta curve shows the model without non-orthogonalities; this is rather close to the curve of the full model and indicates the decoupling of the nonorthogonalities from any long-term temporal effect of the measurement disturbances and instruments.

Maps of the three components of the estimated disturbance fields from the full model as function of Sun incidence angles $\alpha$ (abscissa) and $\beta$ (ordinate) are given in Figs. 7, 8, and 9 for Swarm Alpha, Bravo, and Charlie, respectively. During nominal flight, the Sun incidence angles traverse these plots horizontally from right to left and move up or down in $\beta$ as the orbit plane moves through local time. The Sun-induced disturbance is observed to have temporal characteristics that are observed in the plots as horizontally stretched features, and these are attributed to thermal capacitance: The Suninduced disturbance exhibits characteristic warm-up and cool-down effects, i.e. the disturbance increases when the spacecraft is exposed to the Sun, and decreases when the Sun exposure terminates. The time constants for these effects are up to tens of minutes (corresponding to several tens of degrees in the $\alpha$ angle). This effect is captured 

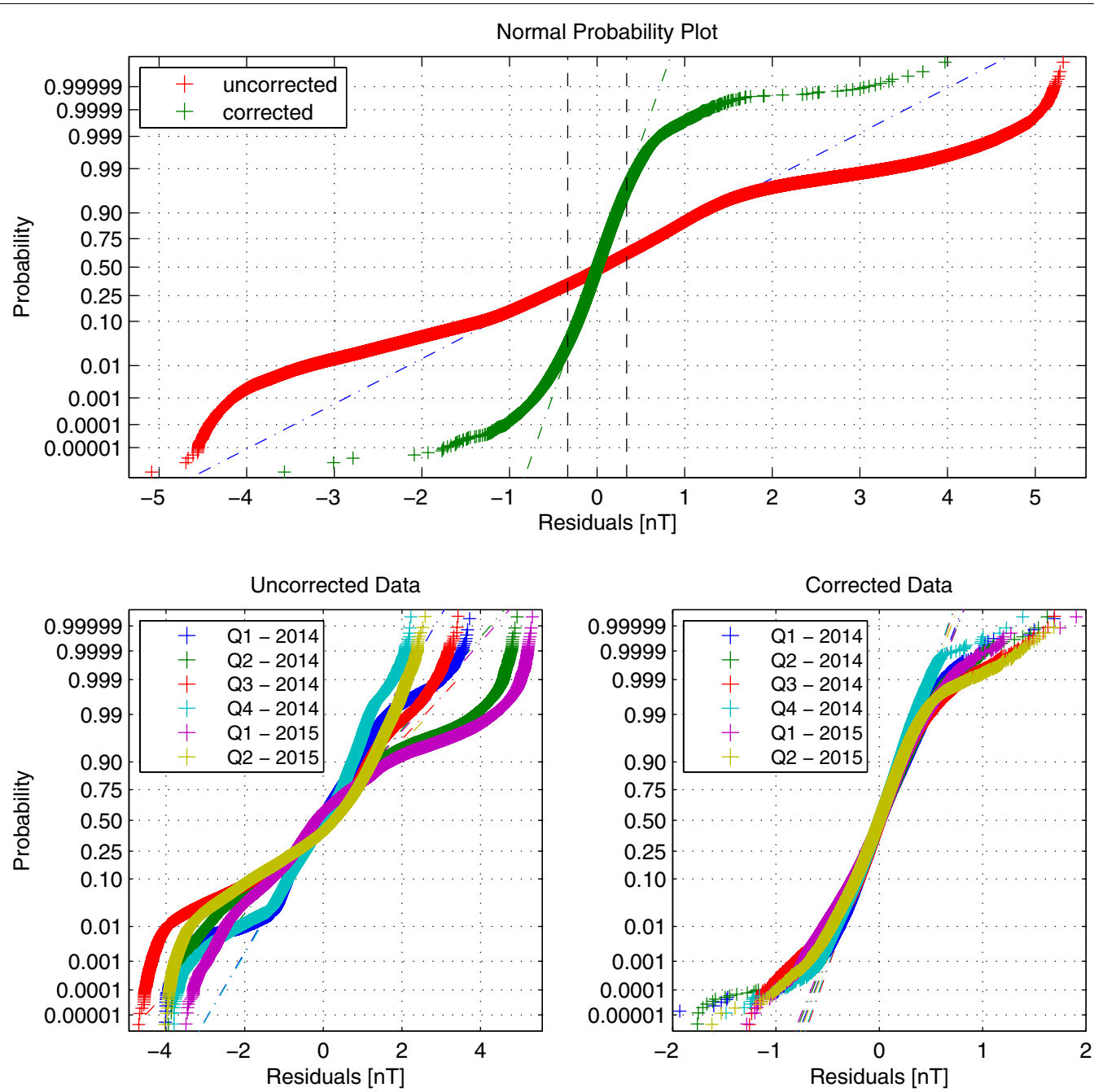

Fig. 5 Normal distribution plots of scalar residuals. Top all data, uncorrected (red), and corrected (green); the limits corresponding to the Huber weights (for corrected data) are shown by the vertical dashed lines. Bottom distributions split into quarters, uncorrected (left), and corrected (right), respectively

Table 3 Estimated values for selected model parameters for all three Swarm satellites

\begin{tabular}{|c|c|c|c|c|c|c|}
\hline \multirow[t]{2}{*}{ Sat } & \multicolumn{2}{|c|}{$\begin{array}{l}\text { Sensitivity/sensor temperature, } s_{T s e n s o r} \\
\left(10^{-6} /{ }^{\circ} \mathrm{C}\right)\end{array}$} & \multicolumn{2}{|c|}{ Sensitivity $/ \beta$ angle, $s_{\beta}\left(10^{-6} / \mathrm{deg}\right)$} & \multicolumn{2}{|c|}{$\begin{array}{l}\text { Non-orthogonalities, } u_{1,2,3} \text { (arc- } \\
\text { seconds) }\end{array}$} \\
\hline & Pre-flight & Adjustment & Pre-flight & Adjustment & Pre-flight & Adjustment \\
\hline \multirow[t]{3}{*}{ Alpha } & 28.5 & 0.616 & - & -0.125 & 102.386 & -0.601 \\
\hline & 28.8 & 0.780 & - & 0 & 217.403 & -3.960 \\
\hline & 28.3 & 0.945 & - & 0.012 & -179.318 & 0.149 \\
\hline \multirow[t]{3}{*}{ Bravo } & 28.3 & 1.168 & - & -0.132 & 350.880 & -0.558 \\
\hline & 29.0 & 1.385 & - & -0.003 & 62.432 & -2.453 \\
\hline & 28.8 & 1.602 & - & -0.198 & -147.060 & 1.608 \\
\hline \multirow[t]{3}{*}{ Charlie } & 27.7 & 1.521 & - & -0.090 & 139.140 & 0.094 \\
\hline & 29.1 & 1.300 & - & -0.038 & -248.890 & 1.042 \\
\hline & 28.4 & 1.076 & - & -0.167 & -109.960 & 0.805 \\
\hline
\end{tabular}

The $n$ T-equivalents of the adjustments in a $50,000 \mathrm{nT}$ ambient field are: STsensor $=10^{-6} /{ }^{\circ} \mathrm{C} \sim 1.25 \mathrm{nT}\left(25^{\circ} \mathrm{C}\right.$ temperature swing $), s_{\beta}=0.1 \times 10^{-6} /$ deg $\sim 0.45 \mathrm{nT}$ $\left( \pm 90^{\circ}\right), u=1$ arc - second $\sim 0.242 \mathrm{nT}$ 
Table 4 Weighted rms values for various models, Swarm Alpha

\begin{tabular}{llc}
\hline Model & Weighted rms (pT) & $\begin{array}{l}\text { Residual power } \\
\text { (normalised) (\%) }\end{array}$ \\
\hline Full model & 168.3 & 100 \\
No $S_{\beta}$ & 176.1 & 107 \\
No $S_{\text {Tsensor }}$ & 181.7 & 116 \\
No non-orthogonalities & 250.2 & 221 \\
No $\delta \vec{B}_{\text {Sun }}$ & 962.6 & 3269 \\
\hline
\end{tabular}

by the spherical harmonic model expansion of $\delta \vec{B}_{\text {Sun }}$ and yields the horizontally stretched features in Figs. 7, 8, and 9. Note also the regions of nightside data (eclipse), the circled areas to the left of the figures, which generally show less disturbance; this is not imposed by the model or any regularisation; rather, it is simply a result of the data itself and thus another indicator of the ability of the model to describe the observed disturbances. The plots also show both the similarities and the differences in $\delta \vec{B}_{\text {Sun }}$ between the three satellites.

\section{Conclusions}

We have established a predominantly empirical model for the calibration and correction of the magnetic vector field measurements of the three Swarm spacecraft. The model is based on detailed studies of the observed scalar residuals between the measurements of the absolute scalar magnetometer, ASM, and the modulus of the measurements of the vector field magnetometer, VFM. The model has proven to be quite robust as more data are incorporated into the estimation of the model

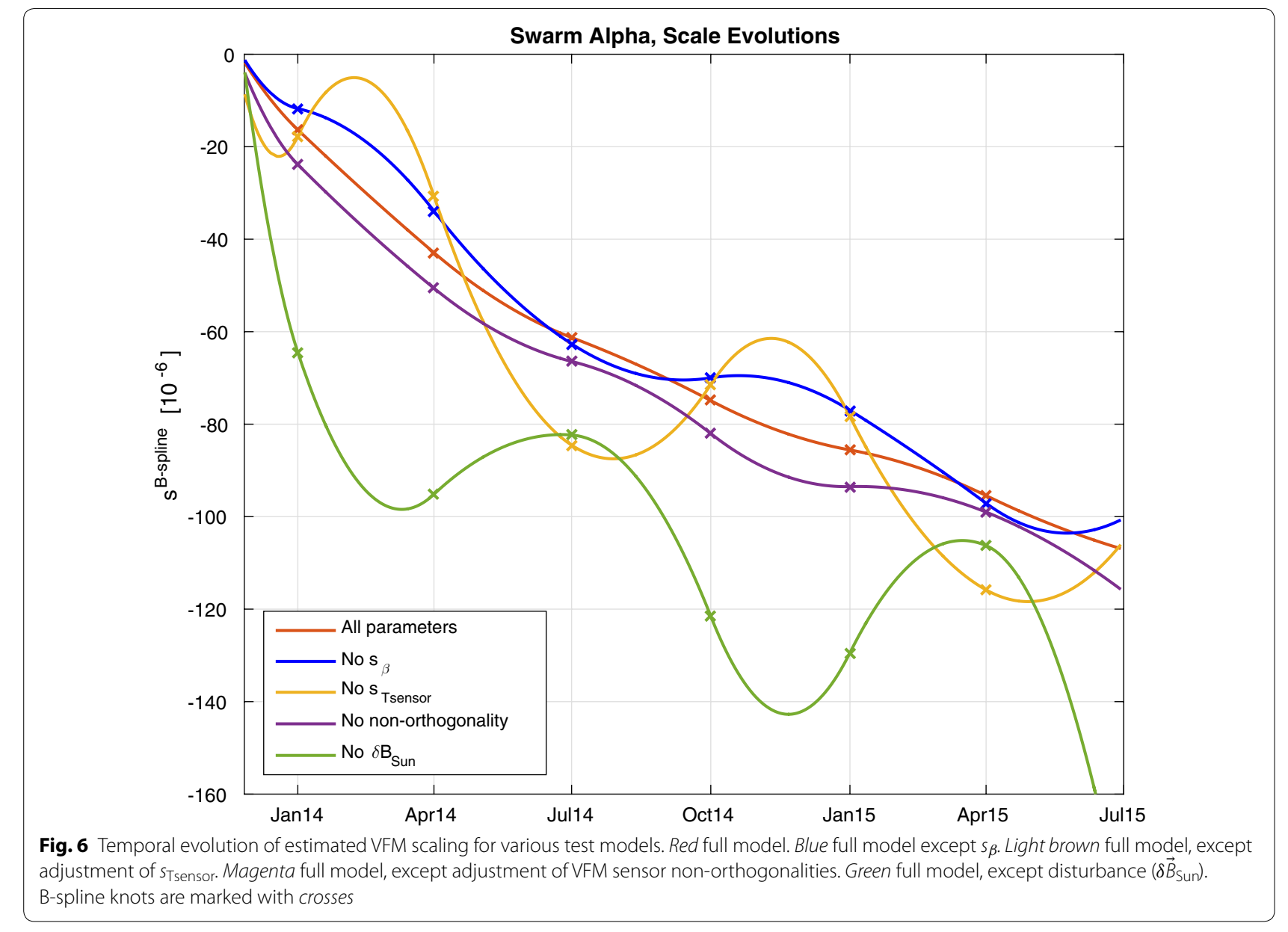



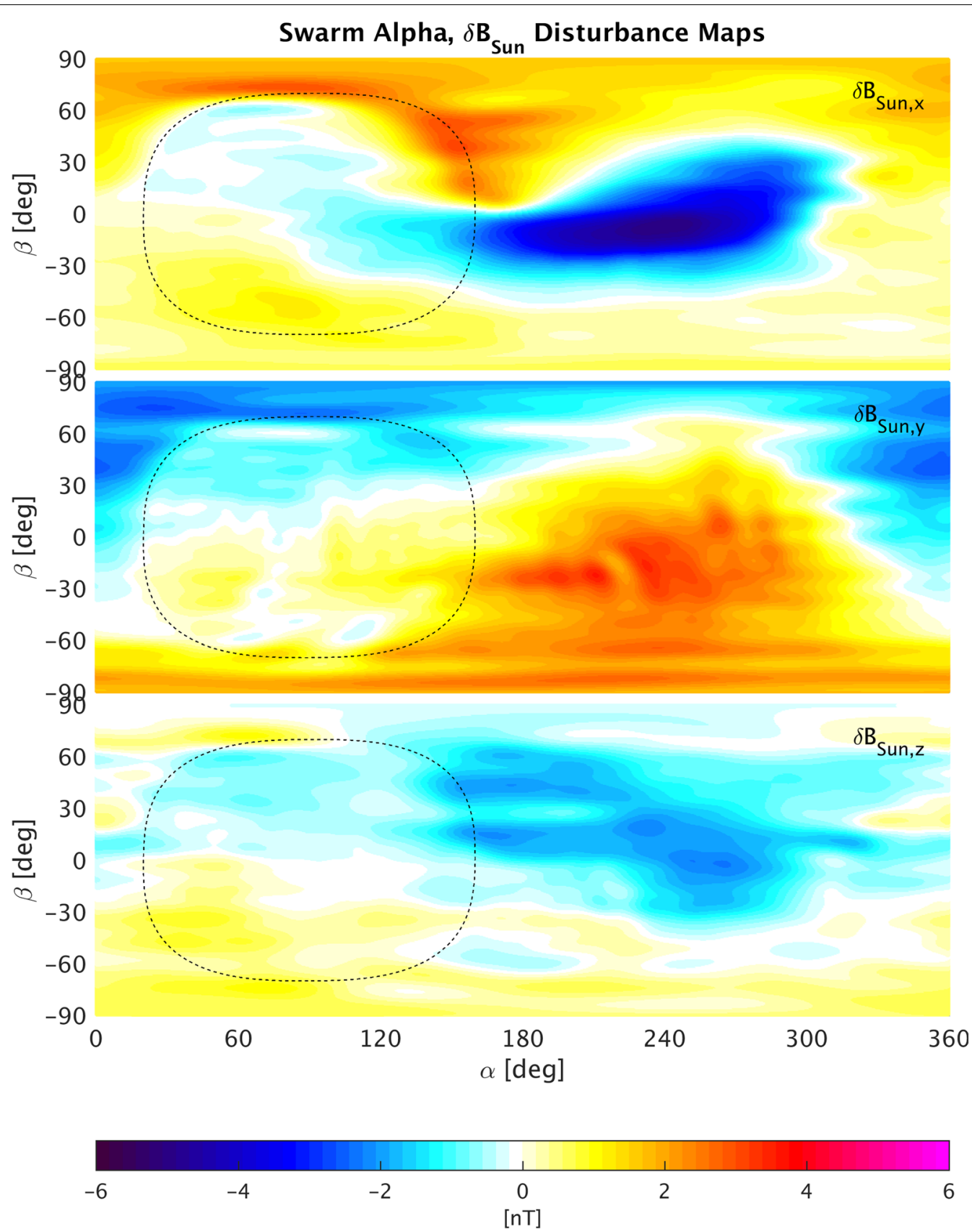

Fig. 7 Maps of estimated $\delta \vec{B}_{\text {Sun }}$ for Swarm Alpha componentwise as function of Sun incident angles $\alpha$ and $\beta$ ( $x$ - and $y$-axes, respectively). The circled area indicates the approximate region of the satellite being in eclipse

parameters, although the ambiguity of determining vector disturbances from a pure scalar calibration affects the estimated correction vectors; these corrections do change slightly (by a few tenths of a nT) as more data are added.

The estimated models reduce the scalar differences between the Swarm magnetometers to generally below
$0.5 \mathrm{nT}$ with rms values well below $200 \mathrm{pT}$ for all three satellites and have been in operational use since April 2015 to produce corrected Swarm Level 1b magnetic field vector data (as of version 0401).

Future evolutions of the model presented here are foreseen to include changing the model of the temporal evolution of the VFM sensitivity from B-splines to 

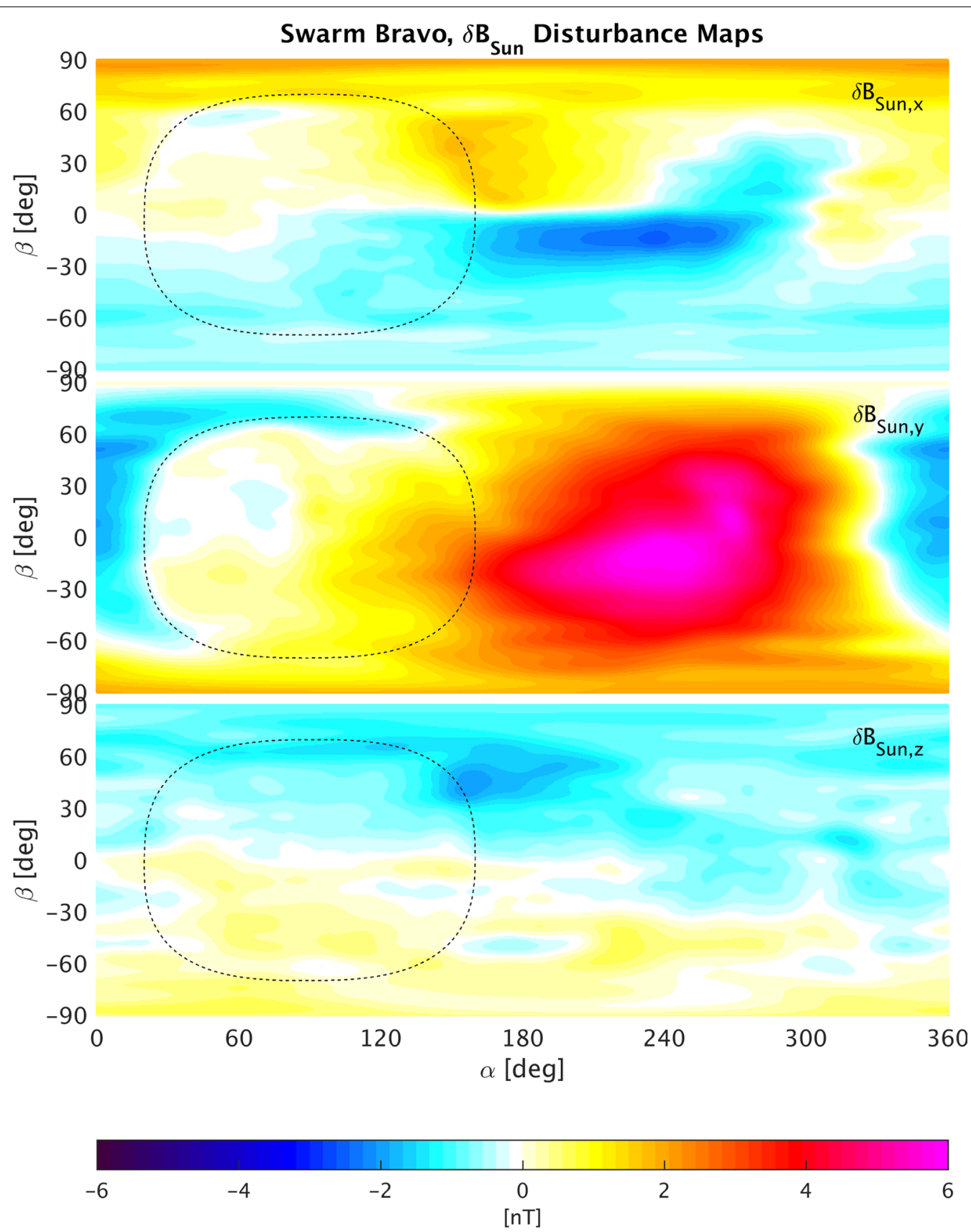

Fig. 8 Maps of estimated $\delta \vec{B}_{\text {Sun }}$ for Swarm Bravo similar to Fig. 7

an exponentially decaying function. Analysis of $\delta \vec{B}_{\text {Sun }}$ also indicates that this vector is generally confined to a few, distinct directions which may be incorporated in future models. Finally, it may be possible to model the effect of the thermal capacitance using appropriate temporal filter functions which would lead to a significant reduction of the number of parameters of the model. 

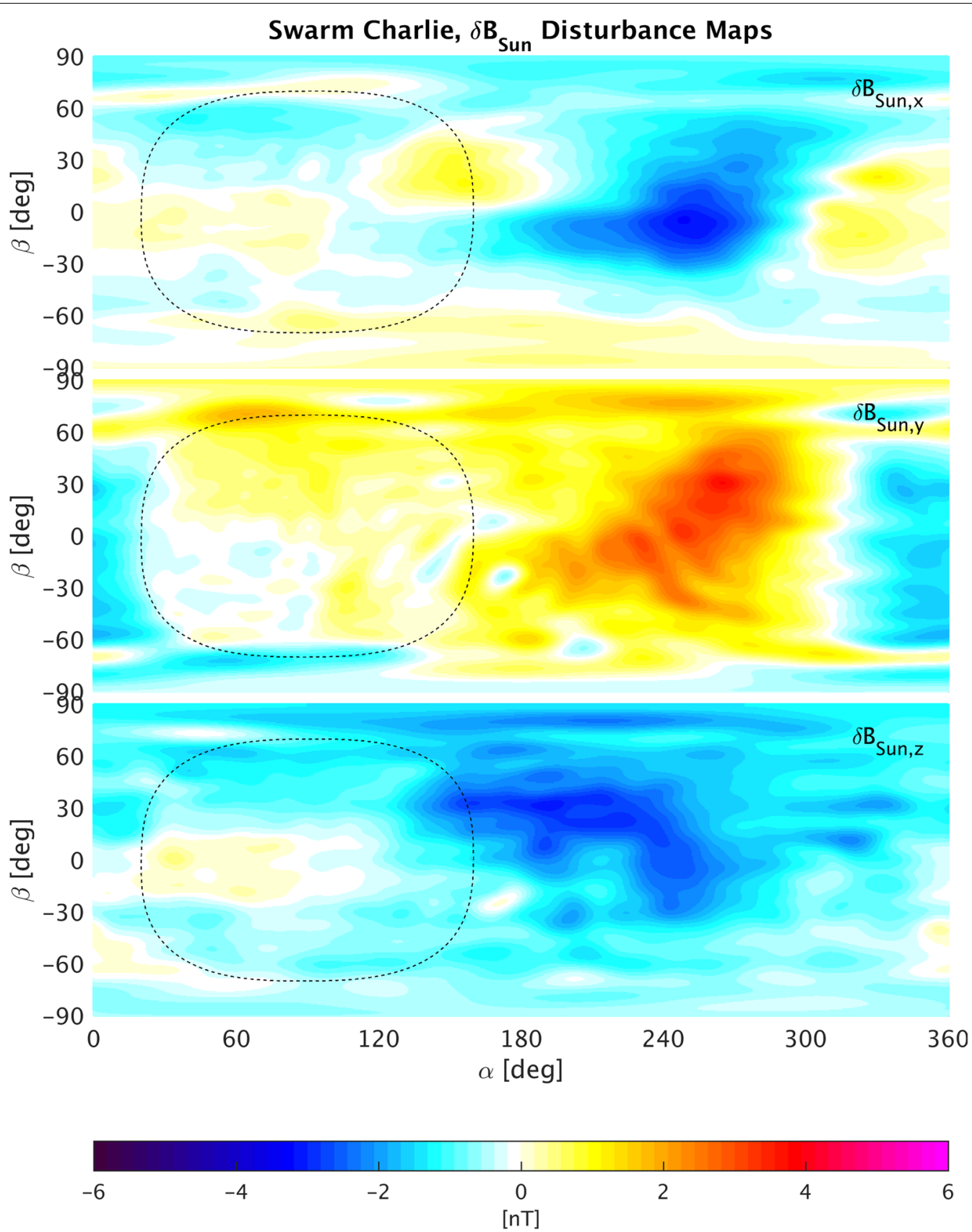

Fig. 9 Maps of estimated $\delta \vec{B}_{\text {Sun }}$ for Swarm Charlie similar to Figs. 7 and 8

Data availability

The estimated disturbance vectors, $\delta \vec{B}_{\text {Sun }}$, are included in the operational Level 1b magnetic Swarm data products as $d B$ sun.
Uncorrected data are available at ftp://swarm-diss. eo.esa.int/Advanced/ (login required, access can be requested via https://earth.esa.int/Swarm). 


\section{Authors' contributions}

LTC carried out the in-flight scalar calibration and characterisation, analysed the results, and led the writing of this manuscript. VL proposed the model for the Sun-induced vector disturbance, $\delta B_{\text {Sun }}$, and made the first estimations using this model. $\mathrm{NiO}$ and CF supported the entire project with many discus sions, suggestions, and source code. All authors read and approved the final manuscript.

\section{Author details}

1 Division of Geomagnetism, DTU Space, Technical University of Denmark, Diplomvej, Kongens Lyngby, Denmark. ${ }^{2}$ National Magnetic Observatory Geomagnetism, Institut de Physique du Globe de Paris, 1 rue Jussieu, Paris, France.

\section{Acknowledgements}

We would like to thank ESA for establishing and providing support to the ASM-VFM Task Force with the aim of investigating the source of the scalar residuals observed in the Swarm magnetic measurements and developing a correction scheme. We would also like to thank this Task Force for its work in characterising the behaviour of the magnetic disturbance and for many fruitful discussions and inputs for this work. In particular, we would like to thank Peter Brauer from the VFM instrument team for detailed discussions on the modelling and on the characteristics of the VFM instruments. Two anonymous reviewers are thanked for their comments that helped to improve the clarity of the manuscript. This paper is the IPGP contribution 3761.

\section{Competing interests}

The authors declare that they have no competing interests.

Received: 29 January 2016 Accepted: 29 June 2016

Published online: 22 July 2016

\section{References}

Friis-Christensen E, Lühr H, Hulot G (2006) Swarm: a constellation to study the Earth's magnetic field. Earth Planets Space 58:351-358

Lesur V, Rother M, Wardinski I, Schachtschneider R, Hamoudi M, Chambodut A (2015) Parent magnetic field models for the IGRF-12 GFZ-candidates. Earth Planets Space. doi:10.1186/s40623-015-0239-6

Olsen N et al (2003) Calibration of the Ørsted vector magnetometer. Earth Planets Space 55:11-18

Tøffner-Clausen L (2015), Swarm Level 1 b processor algorithms. Esa doc. sw-rsdsc-sy-0002, National Space Institute, DTU Space, Copenhagen

Yin F, Lühr H (2011) Recalibration of the CHAMP satellite magnetic field measurements. Meas Sci Technol 22(5):055,101

\section{Submit your manuscript to a SpringerOpen ${ }^{\odot}$ journal and benefit from:}

- Convenient online submission

- Rigorous peer review

- Immediate publication on acceptance

- Open access: articles freely available online

- High visibility within the field

- Retaining the copyright to your article 